\title{
Whole genome analysis of a livestock-associated methicillin-resistant Staphylococcus aureus ST398 isolate from a case of human endocarditis
}

\author{
Maarten J Schijffelen, CH Edwin Boel, Jos AG van Strijp and Ad C Fluit*
}

\begin{abstract}
Background: Recently, a new livestock-associated methicillin-resistant Staphylococcus aureus (MRSA) Sequence Type 398 (ST398) isolate has emerged worldwide. Although there have been reports of invasive disease in humans, MRSA ST398 colonization is much more common in livestock and demonstrates especially high prevalence rates in pigs and calves. The aim of this study was to compare the genome sequence of an ST398 MRSA isolate with other S. aureus genomes in order to identify genetic traits that may explain the success of this particular lineage. Therefore, we determined the whole genome sequence of S0385, an MRSA ST398 isolate from a human case of endocarditis.

Results: The entire genome sequence of $\mathrm{S0385}$ demonstrated considerable accessory genome content differences relative to other $S$. aureus genomes. Several mobile genetic elements that confer antibiotic resistance were identified, including a novel composite of an type V (5C2\&5) Staphylococcal Chromosome Cassette mec (SCCmec) with distinct joining $(J)$ regions. The presence of multiple integrative conjugative elements combined with the absence of a type I restriction and modification system on one of the two vSa islands, could enhance horizontal gene transfer in this strain. The ST398 MRSA isolate carries a unique pathogenicity island which encodes homologues of two excreted virulence factors; staphylococcal complement inhibitor (SCIN) and von Willebrand factor-binding protein (vWbp). However, several virulence factors such as enterotoxins and phage encoded toxins, including Panton-Valentine leukocidin (PVL), were not identified in this isolate.
\end{abstract}

Conclusions: Until now MRSA ST398 isolates did not cause frequent invasive disease in humans, which may be due to the absence of several common virulence factors. However, the proposed enhanced ability of these isolates to acquire mobile elements may lead to the rapid acquisition of determinants which contribute to virulence in human infections.

\section{Background}

Staphylococcus aureus is an important pathogen that can cause a wide variety of hospital- and communityacquired infections. In addition, it is also part of the normal flora in $20-40 \%$ of adult humans [1]. In hospitals throughout the world, antibiotic pressure combined with the adaptive genetic capacity of S. aureus has resulted in the development of lineages with resistance to multiple antimicrobial drugs, including methicillin. Hospital-associated methicillin-resistant $S$. aureus (HA-MRSA) has become a major source of nosocomial infections and is associated with increased morbidity and mortality [2].

*Correspondence: A.C.Fluit@umcutrecht.nl

1 Department of Medical Microbiology, University Medical Centre Utrecht, Heidelberglaan 100, 3508 GA, Utrecht, the Netherlands

Full list of author information is available at the end of the article
However, in the past decade, MRSA has increasingly caused infections and outbreaks outside the hospital setting, particularly among healthy people who lack the known risk factors for MRSA acquisition. These isolates are referred to as community-associated MRSA (CAMRSA) and are genetically distinct from HA-MRSA. Considerable genetic heterogeneity has been observed among CA-MRSA strains, which contrasts with the presence of a few pandemic clones of HA-MRSA [3]. In 2003, a new MRSA lineage, Sequence Type 398 (ST398), emerged in the community [4,5]. ST398 isolates can cause several acute infections including benign skin and soft tissue infections, respiratory tract infections, as well as serious life-threatening conditions such as bacteremia and endocarditis [6-8]. Recent studies have shown that a major risk factor in ST398 human infections is a profes- 
sional relationship with livestock farming, especially with pigs and calves [9-12]. Indeed, MRSA ST398 colonization of these animals is quite common, and overall prevalence rates for pigs are as high as $49 \%$ [13]. This is the first time that such a large MRSA reservoir was identified outside of a hospital environment. The identification of livestock in MRSA ST398 transmission is of considerable concern, especially since livestock is thought to be the source of several emerging antimicrobial-resistant bacteria in the community. Currently, MRSA ST398 has been identified throughout the world, including countries in Europe, North America and Asia [14-18]. Thus, MRSA ST398 represents a distinct lineage that has rapidly spread worldwide and is mainly associated with animal colonization in livestock farming. However, this isolate is also able to colonize and cause invasive disease in humans. Therefore, the entire genome sequence of an ST398 isolate was determined and compared with other $S$. aureus genomes, in order to identify genetic traits that may explain the success of this global lineage.

\section{Results}

The MRSA ST398 genome consists of a circular chromosome of 2,872,582 bp, as well as 3 circular plasmids. The size of the chromosome is comparable to that of other sequenced $S$. aureus strains. We identified 2,699 ORFs, 6 ribosomal RNA operons, and 24 mobile genetic elements, including 12 insertion sequence (IS) elements. The general features of the genome are summarized in Table 1. In total, 37 predicted genes had no significant homologues in public databases. The accessory component of the genome is mainly represented within 12 mobile genetic elements on the chromosome and in the three plasmids.
Most of these genetic elements carry virulence and resistance genes involved in colonization and pathogenesis, and are likely acquired horizontally as indicated by integration at specific loci and/or the presence of recombinase genes, flanking direct repeats, as well as GC content. As observed in other S. aureus genomes, the majority of unique genes are encoded on allotypes of known mobile genetic elements.

A $38 \mathrm{~kb}$ type V (5C2\&5) Staphylococcal Cassette Chromosome mec (SCCmec), which contains the mecA gene that confers methicillin resistance, is inserted at the characteristic chromosomal integration site (3'-end of the orf X gene) of MRSA S0385. Type V SCCmec is characterized by the presence of a $\mathrm{ccrC}$ gene in the ccr gene complex, which encodes a site-specific recombinase that is required for excision and integration of the SCCmec element that is flanked by direct repeats containing the integration site sequence [19]. The S0385 SCCmec element appears to be composed of gene clusters that have been previously described for other SCC elements (Figure 1 and Additional file 1: Table S1). The variable non-essential joining 3 (J3) region harbors a second intact $c c r C$ gene complex directly downstream of orf $X$. This region is similar to the $c c r C$ complex in $\mathrm{SCCHg}$ [20]. Although $\mathrm{SCCHg}$ contains a truncated $c c r C$ gene, it can still be mobilized due to the presence of flanking integration site sequences, while the neighboring type III SCCmec element may provide a recombinase. Similar alternative excision has been described for another MRSA strain carrying a type IV SCCmec with additional integration site sequences [21]. However, type V SCCmec in MRSA S0385 should be considered a composite element because it lacks the additional integration site sequence that is required for

Table 1: General characteristics of the S. aureus S0385 genome

\begin{tabular}{|c|c|c|c|c|}
\hline & Chromosome & pS0385-1 & pS0385-2 & pS0385-3 \\
\hline Size (base pairs) & $2,872,582$ & 5,246 & 4,381 & 3,158 \\
\hline $\mathrm{G}+\mathrm{C}$ content $(\%)$ & $32.9 \%$ & $30.7 \%$ & $31.6 \%$ & $29.0 \%$ \\
\hline Protein-coding genes & 2699 & 4 & 6 & 2 \\
\hline Coding (\%) & $83.5 \%$ & $77.5 \%$ & $78.1 \%$ & $60.7 \%$ \\
\hline Transfer RNA genes & 59 & 0 & 0 & 0 \\
\hline Ribosomal RNA operons & 6 & 0 & 0 & 0 \\
\hline Insertion sequences & 12 & 1 & 0 & 0 \\
\hline Mobile genetic elements & 12 & 0 & 0 & 0 \\
\hline Transposons & 3 & & & \\
\hline Prophages & 2 & & & \\
\hline ScCmec & 1 & & & \\
\hline SaPI & 1 & & & \\
\hline vSa islands & 2 & & & \\
\hline ICE & 3 & & & \\
\hline
\end{tabular}



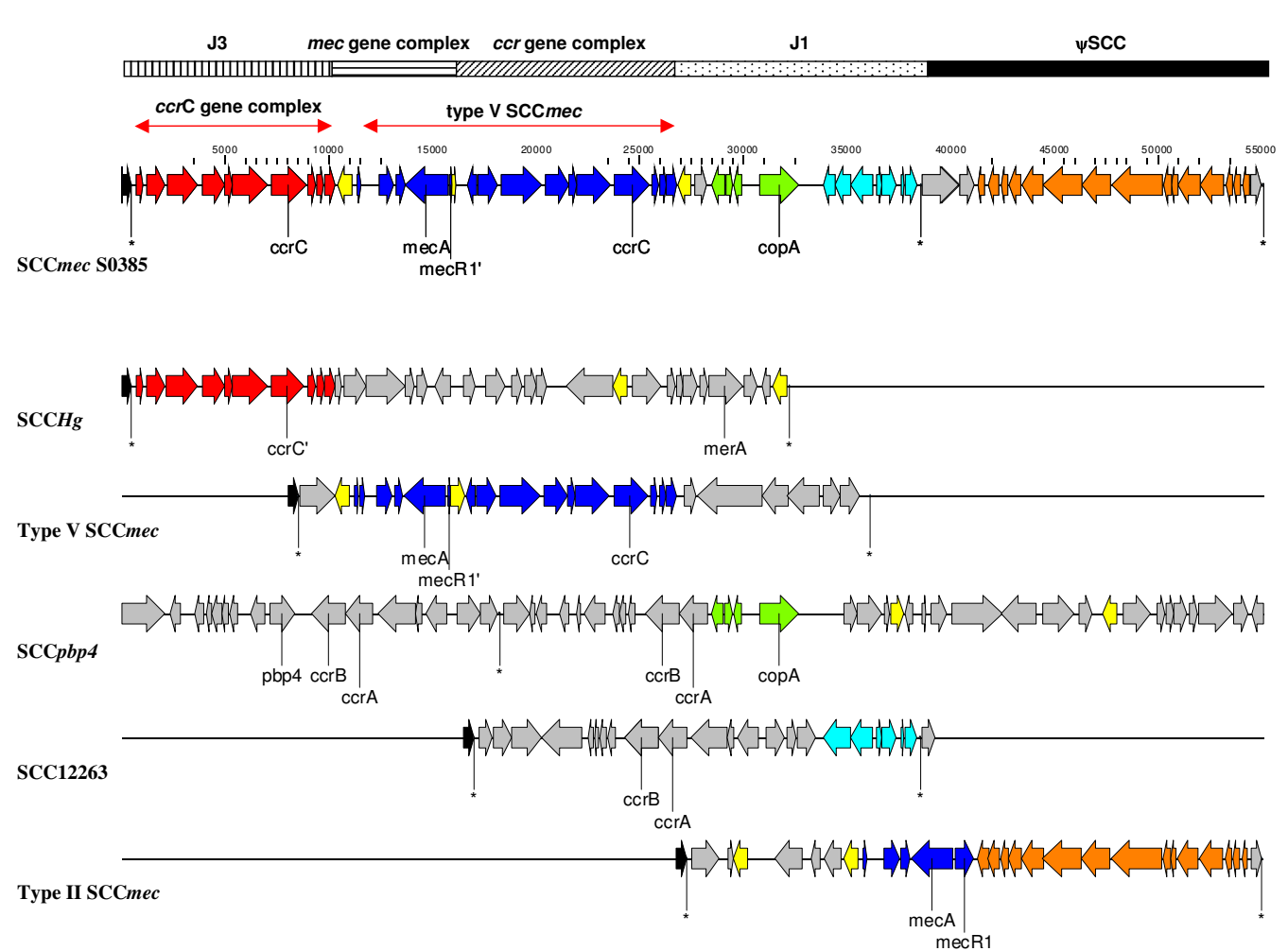

Figure 1 Structure and comparative analysis of SCCmec element of S0385. The SCCmec element of S0385 contains a mec and ccr gene complex with a nearly identical structure compared to type V SCCmec, and two variable non-essential regions that include J1 and J3, the latter which contains a second solitary ccrC gene complex [19]. A pseudo-SCC element ( $\Psi S C C)$ is integrated upstream in tandem with the SCCmec element. Asterisks indicate the three direct repeat containing integration site sequences. The $\$ 0385 \mathrm{SCC}$ mec element appears to be composed of gene clusters that have been previously described for other SCC elements. Shown is an alignment of the 50385 SCCmec element with the other SCC elements (i.e. S. aureus SCCHg, strain85/2082 and type V SCCmec, strain WIS; S. epidermidis SCCpbp4, strain ATCC12228; S. hominis SCC12263, strain GIFU12263 and S. aureus type II SCCMec, strain JCSC6826). Arrows represent ORFs and their direction of transcription. The homologous clusters of ORFs are indicated with similar colors. Red: solitary ccrC gene complex; blue: SCCmec type V mec and ccrC gene complex; green: cluster containing copA gene; aqua: metallo-hydrolase gene complex; orange: integrative conjugative element; yellow: IS431; black: orfX; grey: ORFs with no homology to the other SCC elements shown.

alternative excision of the two elements. In addition, within region J1, the SCCmec element of S0385 carries a copA gene that may confer copper resistance. This gene was previously found only in the SCCpbp4 element of Staphylococcus epidermidis ATCC 1228 [22]. Finally, a truncated metallo-hydrolase gene complex, similar to Staphylococcus hominis SCC12263 was also identified in this isolate.

Type V (5C2\&5) SCCmec elements with a $c c r C$ gene containing J3 region have recently been found in a MRSA ST59 isolate [GenBank: AB462393] and a methicillinresistant Staphylococcus pseudintermedius isolate [GenBank: FJ544922]. Each of these isolates has an integration site sequence at $\operatorname{orf} X$ which is identical to that of the SCCmec element in S0385. However, they possess numerous SNPs in the intergenic region upstream of the first IS431 element and in the final two ORFs of the $c c r C$ complex. Furthermore, the content of the J1 region is unique for all three elements and a transposon is also integrated into the SCCmec of MRSA ST59.

Immediately downstream of SCCmec is a second 16.5 $\mathrm{kb}$ pseudo-SCC element that is integrated into orf X (Figure 1 and Additional file 1: Table S1). Although it is flanked by SCC-specific integration site sequences, this second element lacks any $c c r$ genes suggesting that it depends on the SCCmec encoded recombinase for excision. The pseudo-SCC element carries 20 ORFs of which three show no homology to any known genes and 17 represent a gene cluster with homology to integrative conjugative elements (discussed in more detail below). This gene cluster is also present in the $S$. aureus genomes of COL, USA300 FR3757 and MRSA252, but is always integrated at integration sites not related to an SCC element. Recently, one example of this 17 gene cluster has been described in association with an SCC element. It was integrated into the $\mathrm{J} 1$ region of a type II SCCmec from isolate JCSC6826. However, the gene cluster was inte- 
grated without SCC-specific integration site sequences and lacks the additional three ORFs that flank the pseudo-SCC element in S0385.

Two nearly identical bacteriophages of approximately $47 \mathrm{~kb}, \phi$ Sa6S0385 and $\phi S a 2 S 0385$, have integrated into the chromosome at the lipase gene and a hypothetical protein, respectively. The attachment site and integrase of $\phi$ Sa6S0385 and $\phi S a 2 S 0385$ were similar to those of the $\phi \mathrm{COL}$ and $\phi \mathrm{Sa} 2$ family, respectively. The two phages demonstrated considerable differences at the terminal ends, which include the integrase and endolysin genes. The domains that were common to both bacteriophages had a mosaic structure and consisted mainly of genes encoding phage regulatory, tail and capsule proteins. We were not able to identify any known virulence gene besides NWMN0280, which is a virulence associated gene previously identified in the phages of $S$. aureus Newman. The remarkable presence of two highly homologous phages has only been described once before in the $S$. aureus Newman strain [23].

Three transposons were integrated into the core chromosome. A transposon similar to Tn916 of Enterococcus faecalis carries the tet $(\mathrm{M})$ tetracycline resistance element and a gene cluster required for conjugative transfer. The second transposon, Tn552, encodes an inducible $\beta$-lactamase and its regulator components. Both elements are commonly detected in tetracycline- and $\beta$-lactam-resistant staphylococci, respectively. A third transposon showed similarity with the Tn7 transposon family. Members of this family are usually found in Gram-negative bacteria. However, recently Tn7-like transposons have been reported in Gram-positive bacteria, such as Bacillus cereus and staphylococci [24]. It is unknown which function(s) are encoded by the Tn7-like transposon in $\mathbf{S 0 3 8 5}$ as it lacks typical genes that are known to confer antibiotic resistance or enhance pathogenesis.

Two nearly identical $15 \mathrm{~kb}$ putative mobile genetic elements were integrated into the genome. One was contained in the pseudo-SCC element while the other was integrated into the acyl-coenzyme A ligase gene. The integration appears to result in the duplication of 3 nucleotides. Furthermore, nearly identical elements were also integrated in the USA300 FPR3757, MRSA252 and COL genomes, as well as type II SCCmec from the isolate JCSC6833. The element contains 17 ORFs, which includes proteins with conserved domains related to amidase, ATPase, DNA translocase, a replication initiation factor and a gene with a frame-shift encoding an integrase. Frame-shifts among integrase genes have been previously reported [25]. These frame-shifts are reversible and are thought to contribute to the regulation of the transfer of the genetic element. Nine of the 17 proteins demonstrate similarity with those found in integrative and conjugative elements from Bacillus subtilis (ICEBs1) and Listeria monocytogenes (ICELm1) (Figure 2 and Additional file 1: Table S2). Indeed, the circularized form of the element was detected by PCR (data not shown) suggesting that this element can be excised from the chromosome and may be transferred by conjugation with subsequent integration into the recipient. We named these elements, ICESa1A and ICESa1B. A third similarly organized element containing a cluster of genes homologous to ICESa1 was integrated into a conserved hypothetical protein and named, ICESa2 (Figure 2 and Additional file 1: Table S2). However, we did not identify any known virulence genes in these three elements.

A novel staphylococcal pathogenicity island (SaPI), designated as SaPI-S0385, was also identified. This SaPI was integrated into the same conserved $20 \mathrm{bp}$ attachment site as SaPIbov (ET3) and composed at the 5'-end of sequences homologous to SaPIbov and SaPI5 (USA300 FPR3757). These sequences encode genes involved in transcription, replication and packaging (Figure 3 and Additional file 1: Table S3). A unique region at the 3'-end of SaPI-S0385 was identified to encode two putative extracellular proteins with similarity to staphylococcal complement inhibitor (SCIN) and von Willebrand factorbinding protein $(\mathrm{vWbp})$, respectively. Both proteins also have a conserved homologue in the core genome of S0385. The other genes identified on SaPI-S0385 were a putative primase and hypothetical phage related genes.

Novel allelic variants of $\nu \mathrm{Sa} \alpha$ and $\nu \mathrm{Sa} \beta$ islands were also identified. Both of these $v$ Sa islands are present in all $S$. aureus genomes and each is integrated at a conserved position in the chromosome. There are considerable differences between the strains with respect to the genetic content of the $v \mathrm{Sa}$ islands. However, a key element that has been observed in each $v \mathrm{Sa}$ island is a type I restriction-modification (R-M) system. The presence of two R$M$ systems effectively prevents horizontal gene transfer into the genome and limits DNA transfer between $S$. aureus isolates to those of the same lineage [26]. Although both types of $v \mathrm{Sa}$ islands are present in the S0385 genome, only $v$ Sa $\alpha$ harbors a type I R-M system, which has a unique $h s d S$ gene that encodes the $S$ subunit which determines the sequence specificity of type I R-M systems. The $v \mathrm{Sa} \alpha$ island also contains a staphylococcal superantigen-like (SSL) family of exotoxins as well as a family of lipoproteins. The proteins of the SLL family may play a role in innate immunity evasion, as SSL5 inhibits Pselectin-mediated neutrophil rolling and SSL7 binds IgA and complement factor C5 $[27,28]$. To our knowledge, a function for the family of lipoproteins has not yet been described. The $v \mathrm{Sa} \beta$ island harbors a hyaluronidase gene cluster that was also present in the bovine derived $S$. aureus strain, ET3. This cluster encodes a variant of the hyaluronidase encoded on the core genome of $S$. aureus. Hyaluronidase is a proposed virulence factor that is capa- 


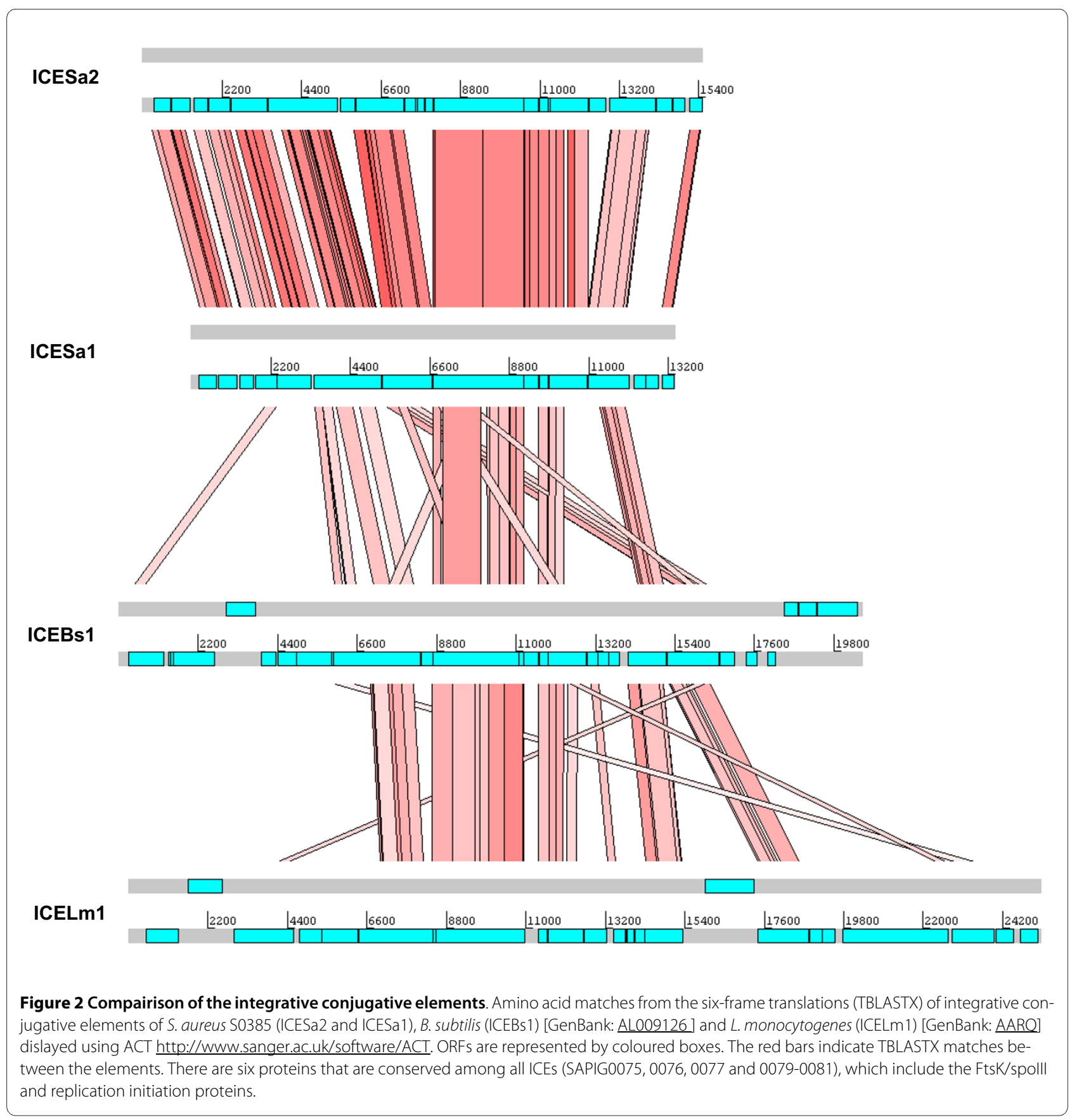

ble of degrading hyaluronic acid, a major component of the extracellular matrix in connective tissue, and thereby contributes to subcutaneous bacterial spread from the initial site of infection. Alternatively, the degradation products of hyaluronic acid may serve as a supply of nutrients for the organism $[29,30]$. The $v \mathrm{Sa} \beta$ island lacks enterotoxins, serine proteases, lantibiotic biosynthesis proteins and LukE/D hemolysins.

The genome of S0385 contained three plasmids. A tetracycline resistance determinant tet $(\mathrm{K})$ is carried on plasmid pS0385-1, which is highly similar to pUSA02 from $S$. aureus USA300 FRP3757. A second plasmid carries a str gene which confers streptomycin resistance. Finally, a small cryptic plasmid was also identified (Table 1).

\section{Discussion}

Analysis of the genome of $S$. aureus strain S0385, a member of the livestock-associated MRSA ST398 lineage, showed considerable differences relative to other $S$. aureus genome sequences. The majority of these differences were identified in unique or novel allotypes of mobile genetic elements. This finding stresses the impor- 


\section{SaPIbov (ET3)}

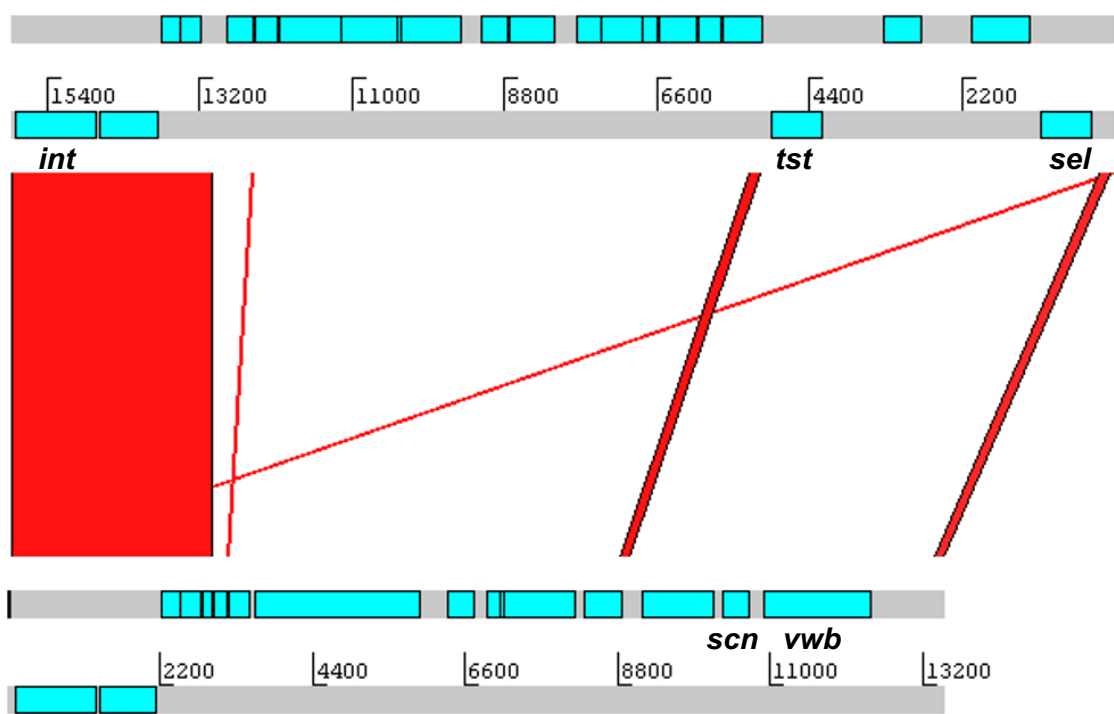

\section{SaPlpig (S0385)}

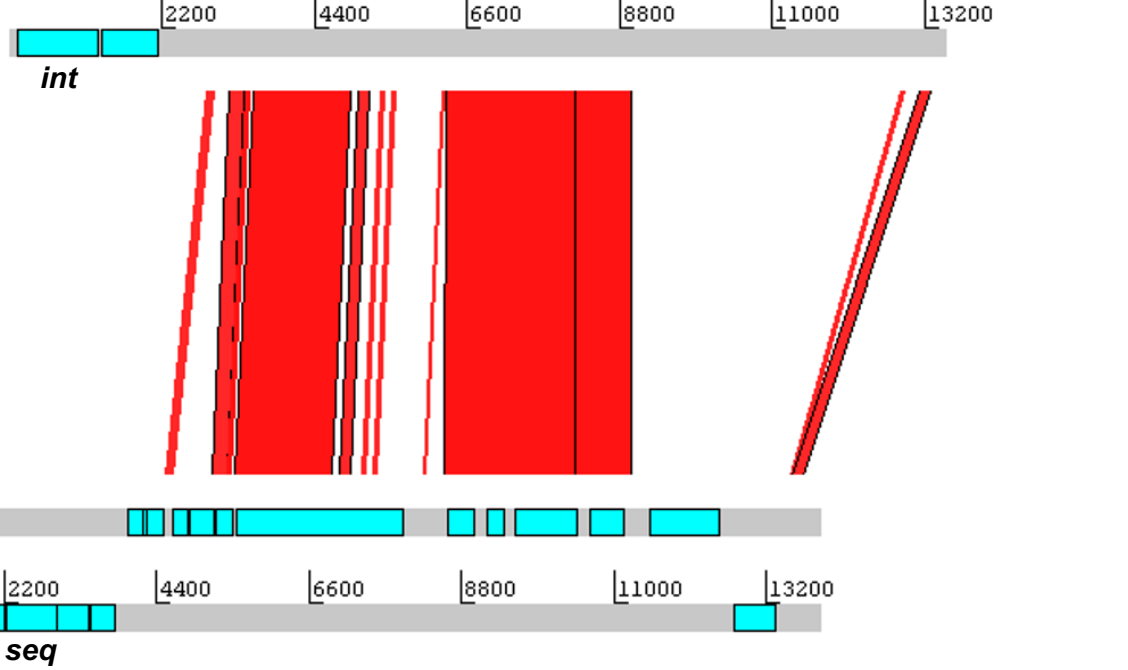

Figure 3 Compairison of the SaPI. Nucleotide matches of SaPIbov, SaPI-S0385 and SaPI5 of S. aureus ET3, S0385 and USA300 FPR3757, respectivly dislayed using ACT. ORFs are represented by coloured boxes. The red bars indicate BLASTN matches between the elements, which mainly concern regions encoding genes that are essential for SAPI replication. The unique region in SaPI-S0385 contains the scn and vwb genes.

tance of horizontal gene transfer in bacterial genome remodeling, as previously reported for other sequenced $S$. aureus genomes. Many of these mobile genetic elements harbor determinants for virulence and antimicrobial resistance which may allow the bacterium to adapt to new niches. It should be noted that some mobile elements such as SaPI-S0385, ICESa1, ICESa2, and phageSa6 do not appear to be present in the majority of 6 spa-types of MRSA ST398, as determined by PCR analysis for specific genes within these elements. However, the tet $(\mathrm{M})$ transposon, vSa encoded hyaluronidase, plasmid encoded streptomycin resistance and type V SCCmec encoded $\operatorname{ccr} C$ were identified in the majority of these isolates (Additional file 1: Table S4).

The presence of 5 different antimicrobial resistance elements, either integrated into the chromosome or located on plasmids, may reflect the relatively high antibiotic pressure in livestock farming. Two tetracycline resistance determinants, tet(M) on Tn916 and tet(K) on pS0385-1, were present in this MRSA isolate. The presence of two of these determinants may confer a higher level of resistance to tetracycline. Tetracycline resistance is most likely responsible for the selection of ST398 isolates. Therapeutic treatment of pigs with oxytetracycline and the treatment of a complete flock with prophylactic oxytetracycline are both common practices in pig farming [17]. Furthermore, a novel type V SCCmec, which encodes methicillin resistance, and harbors two distinct ccrC genes as well as a unique J1 region, was also identified. Recent reports have identified type V SCCmec elements in CA-MRSA ST59 and S. pseudintermedius that harbor two $c c r C$ genes [31,32]. The $c c r C$ encoded recom- 
binase is required for the excision of SCC elements flanked by characteristic integration site sequences from the chromosome, and insertion into another chromosome. Integration site sequences were not found in the sequences between the $2 \mathrm{ccrC}$ genes, suggesting that it is a single SCC element. The presence of two $c c r C$ genes in a single element may enhance recombinase production resulting in an increase in the rate of SCCmec excision, since over expression of $c c r A / B$ leads to increased excision of SCCmec elements [33]. Increased cassette chromosome recombinase $\mathrm{C}$ production may lead to an increased probability of SCCmec transfer between staphylococci. In addition, SCCmec in S0385 carries a putative copper resistance gene complex that is also present in the SCC element of S. epidermidis ATCC 1228. Copper is commonly used as a growth enhancer in pig farming [34]. Therefore, the presence of copper resistance genes may contribute to bacterial survival in this niche. The distinct genetic organization of SCCmec in S0385 is most likely derived from a complex series of recombination and rearrangement processes.

S. aureus's acquisition of DNA from other bacterial species is not very common, which is illustrated by the limited number of $S$. aureus lineages that have acquired SCCmec or vanA transposon resulting in MRSA and vancomycin-resistant $S$. aureus, respectively $[35,36]$. Although $S$. aureus has features that prevent foreign DNA acquisition, some strains appear to be more prone to serve as recipients of DNA transfer than others. In S0385, two potential mechanisms that may increase the transfer and acquisition of foreign DNA were identified. One factor is the absence of a type I R-M system on $v \mathrm{Sa} \beta$. Other sequenced strains possess an R-M system on both $v \mathrm{Sa} \alpha$ and $\beta$ islands. Restriction-modification systems have been postulated to be one of the key elements that prevent foreign DNA integration into the host genome by degradation of DNA that is not methylated by the R-M system's modification enzyme. Indeed, strains that harbor a dysfunctional type I R-M system are significantly more prone to accept foreign DNA via conjugation [37].

Another factor that may influence the acquisition of foreign DNA is the presence of three ICEs in the S0385 genome. One of these ICEs is present in a pseudo-SCC element flanked by integration site sequences and integrated into orf X. ICE encoded proteins form a type IV secretion-like system (T4SLS), which is a multi-protein complex that can hydrolyze the peptidoglycan layer of the cell-wall and transfer single-stranded DNA through both the cell wall and cell membrane. This DNA transfer model supports the hypothesis that ICE-encoded secretion systems are involved in conjugative DNA transport, which has been previously demonstrated for the PIP501 plasmid in gram-positive bacteria [38]. However, homologues of the transfer genes (tra-genes) of pIP501 could not be identified in the S0385 isolate. Alternatively, T4SLS may be involved in protein transport across the cell-wall of the bacterium. The presence of an ICE may have contributed to the acquisition of SCCmec by S0385. In addition, the ICE may also contribute to further transmission of SCCmec and also enhance the transfer of other mobile elements into new hosts. The tremendous diversity of resistance patterns observed among ST398 isolates suggests that they are able to horizontally acquire foreign DNA quite easily under antibiotic pressure [39].

The existence of $S$. aureus lineages with a broad host range, such as ST398, suggests that the bacterial genome can adapt to the host by acquiring specific (virulence) genes or altering gene expression. A recent publication regarding the bovine $S$. aureus ET3 genome suggested that the adaptation of some core genes and acquisition of SaPIbov containing genes that encode TSST- 1 as well as a variant of enterotoxin $C$, contributed to host and tissue specificity [40]. In S0385 we identified SaPI-S0385, which harbors homologues of the $s c n$ and $\nu w b$ genes, both of which encode exoproteins (Additional file 1: Table S3). $\mathrm{SCIN}$, a product of $s c n$ encoded on $\phi \mathrm{Sa} 3$, inhibits complement activation via the classical, alternative and lectin pathways by blocking both the C4b2a and C3bBb C3convertases. It is human-specific as it does not inhibit complement activation in other species, including pigs [41]. SCIN encoded on SaPI-S0385 may demonstrate a broader host range or possibly be pig-specific, which would enable ST398 isolates to inhibit complement activation in their current animal hosts. The $v w b$ homologue on SaPI-S0385 encodes a protein similar to vWbp which binds von Willebrand factor (vWf) [42]. The multifunctional vWf protein plays an important role in hemostasis as it mediates platelet adhesion and aggregation to exposed subendothelium and also binds and stabilizes coagulation factor VIII. The vWbp not only interacts with vWf, but also binds and activates prothrombin without cleaving it, which is the normal pathway for prothrombin activation [43]. The resulting complex can cleave fibrinogen and the resulting fibrin then contributes to the clotting process. Thus, vWbp contributes to clotting via two independent mechanisms. In addition, surface-bound $S$. aureus protein A also interacts with vWf. The presence of both surface bound and soluble proteins that bind vWf may increase the ability of $S$. aureus to adhere at sites of vascular damage, thereby helping the bacterium to gain entry into the host and cause infections. The vWbp encoded in the core genome shows some host-specificity, however both human and porcine serums exhibit comparable coagulation rates after the addition of vWbp [44]. The contribution of SaPI-encoded vWbp is not completely understood. However the presence of a second $\nu w b$ gene may increase clotting activity or help broaden host specificity. 
Interestingly, the MRSA ST398 lineage, which has successfully colonized livestock around the world, seems to lack a large number of well known virulence factors such as enterotoxins and other phage encoded toxins. These results have been confirmed by a recent study that genotyped 54 MRSA ST398 pig isolates using a diagnostic DNA microarray that includes virulence genes and microbial surface components that recognize adhesive matrix molecules [39]. Nevertheless, we identified several additional genes that encode for potential virulence factors such as hyaluronidase, SCIN and vWbp.

\section{Conclusions}

The absence of virulence factors may explain why MRSA ST398 isolates have caused relatively little disease until now. However, the hypothesized enhanced ability of MRSA ST398 to acquire mobile genetic elements may also result in the uptake of mobile elements that encode virulence genes. For example, the first Panton-Valentine Leukocidin (PVL)-positive MRSA ST398 isolates have recently been reported $[9,16,45]$. Considering the vast and increasing animal and human reservoirs, we believe it will only be a matter of time before more of these isolates acquire mobile genetic elements that carry virulence factors which will increase virulence in the human host.

\section{Methods}

\section{Bacterial strain and patient characteristics}

Isolate S0385, an MRSA ST398 SCCmec type V, was obtained in 2006 from a blood culture of a 63 year old female patient with endocarditis of the mitral valve proven by detection of vegetations and an abscess visible on a transoesophageal echocardiogram. The patient had a history of renal transplantation and was treated with immunosuppressive therapy. Antimicrobial susceptibility testing showed that isolate S0385 was resistant to methicillin, ciprofloxacin and tetracycline. The isolate had spa-type t011 and was non-typeable using Pulsed Field Gel Electrophoresis with SmaI, as had been previously observed with MRSA ST398 [7].

\section{Genome sequencing and assembly}

DNA from isolate S0385 was sequenced using pyrophosphate sequencing technology to 16 fold coverage by the 454 group of Roche Applied Sciences. Resulting reads were assembled into contigs and were placed into the correct order and direction using the USA300 FR3757 genome as a scaffold. All gaps between contigs were closed by conventional PCR using primers based on the sequence of flanking contigs followed by sequencing of the products. Several sequences (i.e. insertion elements and rRNA operons) were present more than once in the genome. Each copy of less than $1600 \mathrm{bp}$ in size and all rRNA operons were entirely re-sequenced using conven- tional PCR and unique flanking primers to detect any polymorphisms. Larger sequences were not sequenced individually due to their length and the fact that only single nucleotide polymorphisms were expected.

Annotation was performed using the Annotation Engine, a prokaryotic annotation pipeline developed at the J. Craig Venter Institute (Rockville, MD, USA). A detailed description is available at http://www.jcvi.org/ cms/research/projects/annotation-service/overview/. In short, the prediction of open reading frames (ORFs) and the identification of structural, tRNA, and tmRNA genes were conducted using Glimmer, tRNAscan-SE, BLAST and the Rfam database. Annotation was performed with BER.BLAST and HMMER against a variety of databases. The genome sequence and annotation of S0385 are deposited under the accession numbers [EMBL:AM990992, EMBL:AM990993, EMBL:서990994 and EMBL:AM990995].

\section{Comparative genome analysis}

DNA comparisons were performed using Kodon (Applied Maths, Gent, Belgium). The following S. aureus genomes and corresponding accession numbers were used for comparisons: USA300 FPR3757 [GenBank:CP000255], MRSA252 [GenBank:BX571856], MW2 [GenBank:BA000033], MSSA476 [GenBank:BX571857], N315 [GenBank:BA000018], NCTC8325 [GenBank:CP000253], COL [GenBank:CP000046], Mu50 [GenBank:BA000017], JH1 [GenBank:CP000736], ET3-1 [GenBank:AJ938182] and Newman [GenBank:AP009351].

\section{Detection of virulence and resistance genes}

Seven MRSA isolates were obtained by the Faculty of Veterinary Medicine, Utrecht University, the Netherlands. All isolates were subjected to MLST, spa-typing and PCR-directed SCCmec typing as described previously [17]. The detection of the selected genes encoded by mobile genetic elements was performed by PCR. The following primer pairs were designed and used: SaPI-S0385 int (intSaPI_Fw 5'-TCCACTCTTTGATGAGTGCG CCA; intSaPI_Rv 5'-AGCGCAACAAGACGCATCTACA), $v w b$ (vwb_Fw 5'-TGGGAGCGTTGTGTGCTTCAC; vwb_Rv 5'-CCTGTTCCGTTGTTCC CACCACC) scn (scn_Fw 5'-GCTATTGGTGTAGCTGCGTCGTCA; scn_Rv 5'- TGTGAAGCACACAACGCTCCCA); phageSa2 int (intSa2_Fw 5'-TCAAGTAACCCGTCAACTCGGAGA; intSa2_Rv 5'-TGAACCCTCTGTCAACATAGCTCGAA); phageSa6 int (intSa6_Fw 5'- GGCGATTTTTCTTCTTGAACCTGCGG; intSa6_Rv 5'-GGCGATTTTTC TTCTTGAACCTGCGG); ICESa1 int (intICE1_Fw 5'ACGGGTT CGTGCCTCACACA; intICE1_Rv 5'GCGTGGCAAAT TAGACGTAGGGGC) ICESa2 int (intICE2_Fw 5'-TGCTTCATTCGTGGACGCTGAT; 
intICE2_Rv 5'-AAGATATGCGCCGAGGTGGAAAAA); vSaß hyl (hyl_Fw 5'-TCTGCCACTGGTAAAGCTCGCA; $h y l \_$Rv 5'-CTGCACCGGTGTGCCAACCT) hsdS (hsdS Fw 5' GCTGGTGCATTACCTGTGACAAATGC; hsdS Rv 5'-ATGGTGCAAAATGGGGGCAGT); tet(M) (tetM_Fw 5'-GACGACGGGGCTGGCAAACA; tetM_rv 5'GCCGCCAAATCCTTTCTGGGCT); tet(K) (tetK_Fw 5' GCCCACCAGAAAACAAACCAAGCA; tetK_Rv 5'AGGATCTGCTGCATTCCCTTCACT); str (str_Fw 5'TGCTCTCGAGGGTTCAAGAACTAATGA; str_Rv 5'ACACCCTTTGCTACATACGTTGAGAC).

\section{Additional material}

Additional file 1 Supplementary tables. Table S1 - ORFs in SCCmec from S0385. Table S2 - ORFs in ICESa1 from S0385. Table S3 - ORFs in SAPI from S0385. Table $\$ 4$ - Detection of genes encoded by mobile genetic elements.

\section{Authors' contributions}

MJS: editing and assembly of sequencing data, annotation and bioinformatic analyses of the sequence and also writing of the manuscript. CHEB: bioinformatics analyses and critical revising of the manuscript; JAGS: conception and design of the study and critical revising of the manuscript; ACF: conception and design of the study, editing and assembly of sequencing data, annotation and bioinformatic analyses as well as editing of the manuscript. All of the authors have read and approved the final version of this manuscript.

\section{Acknowledgements}

The authors wish to thank H. Lunstroo (Roche Diagnostics, Netherlands) and Dr. A. Mausolt (Roche Applied Sciences, Germany) for their support. This work was funded in part by a grant from the Dutch Ministry of Agriculture, Nature and Food Quality. The sponsor did not have any role in design and conduct of the study; the collection, management, analysis of the data; or preparation, review or approval of the manuscript.

\section{Author Details}

Department of Medical Microbiology, University Medical Centre Utrecht, Heidelberglaan 100, 3508 GA, Utrecht, the Netherlands

Received: 1 December 2008 Accepted: 14 June 2010

Published: 14 June 2010

\section{References}

1. Williams REO: Healthy carriage of Staphylococcus aureus: its prevalence and importance. Microbiol Mol Biol Rev 1963, 27:56-71.

2. Gould IM: The clinical significance of methicillin-resistant Staphylococcus aureus. J Hosp Infect 2005, 61:277-282.

3. Vandenesch F, Naimi T, Enright MC, Lina G, Nimmo GR, Heffernan H, Liassine N, Bes M, Greenland T, Reverdy ME, Etienne J: Communityacquired methicillin-resistant Staphylococcus aureus carrying PantonValentine leukocidin genes: worldwide emergence. Emerg Infect Dis 2003, 9:978-984

4. Voss A, Loeffen F, Bakker J, Klaassen C, Wulf M: Methicillin-resistant Staphylococcus aureus in Pig Farming. Emerg Infect Dis 2005, 11:1965-1966

5. Witte W, Strommenger B, Stanek C, Cuny C: Methicillin-resistant Staphylococcus aureus ST398 in humans and animals, Central Europe. Emerg Infect Dis 2007, 13:255-258.

6. Huijsdens XW, van Dijke BJ, Spalburg E, van Santen-Verheuvel MG, Heck MEOC, Pluister GN, Voss A, Wannet WJB, de Neeling AJ: Communityacquired MRSA and pig-farming. Ann Clin Microbiol Antimicrob 2006, $5: 26$.

7. Ekkelenkamp MB, Sekkat M, Carpaij N, Troelstra A, Bonten MJ: [Endocarditis due to methicillin-resistant Staphylococcus aureus originating from pigs]. Ned Tijdschr Geneeskd 2006, 150:2442-2447. Dutch
8. Declercq P, Petre D, Gordts B, Voss A: Complicated community-acquired soft tissue infection by MRSA from porcine origin. Infection 2008, 36:590-592

9. van Loo I, Huijsdens X, Tiemersma E, de Neeling A, van de SandeBruinsma, Beaujean D, Voss A, Kluytmans J: Emergence of methicillinresistant Staphylococcus aureus of animal origin in humans. Emerg Infect Dis 2007, 13:1834-1839.

10. Wulf M, van Nes A, Eikelenboom-Boskamp A, de Vries J, Melchers W, Klaassen C, Voss A: Methicillin-resistant Staphylococcus aureus in veterinary doctors and students, the Netherlands. Emerg Infect Dis 2006, 12:1939-1941

11. Wulf MWH, Sørum M, van Nes A, Skov R, Melchers WJG, Klaassen CHW Voss A: Prevalence of methicillin-resistant Staphylococcus aureus among veterinarians: an international study. Clin Microbiol Infect 2008, $14: 29-34$.

12. Lewis HC, Molbak K, Reese C, Aarestrup FM, Selchau M, Sorum M, Skov RL: Pigs as source of methicillin-resistant Staphylococcus aureus CC398 infections in humans, Denmark. Emerg Infect Dis 2008, 14:1383-1389.

13. de Neeling AJ, van den Broek MJM, Spalburg EC, van Santen-Verheuvel MG, Dam-Deisz WDC, Boshuizen HC, van de Giessen AW, van Duijkeren E, Huijsdens XW: High prevalence of methicillin resistant Staphylococcus aureus in pigs. Vet Microbiol 2007, 122:366-372.

14. Armand-Lefevre L, Ruimy R, Andremont A: Clonal comparison of Staphylococcus aureus isolates from healthy pig farmers, human controls, and pigs. Emerg Infect Dis 2005, 11:711-714.

15. Khanna T, Friendship R, Dewey C, Weese JS: Methicillin resistant Staphylococcus aureus colonization in pigs and pig farmers. Vet Microbiol 2008, 128:298-303

16. Yu F, Chen Z, Liu C, Zhang X, Lin X, Chi S, Zhou T, Chen Z, Chen X: Prevalence of Staphylococcus aureus carrying Panton-Valentine leukocidin genes among isolates from hospitalised patients in China. Clin Microbiol Infect 2008, 14:381-384

17. van Duijkeren E, Ikawaty R, Broekhuizen-Stins MJ, Jansen MD, Spalburg EC, de Neeling AJ, Allaart JG, van Nes A, Wagenaar JA, Fluit AC: Transmission of methicillin-resistant Staphylococcus aureus strains between different kinds of pig farms. Vet Microbiol 2008, 126:383-389.

18. Smith TC, Male MJ, Harper AL, Kroeger JS, Tinkler GP, Moritz ED, Capuano AW, Herwaldt LA, Diekema DJ: Methicillin-resistant Staphylococcus aureus (MRSA) strain ST398 is present in midwestern U.S. swine and swine workers. PLOS ONE 2008, 4:e4258.

19. International Working Group on the Classification of Staphylococcal Cassette Chromosome Elements (IWG-SCC): Classification of staphylococcal cassette chromosome mec ( $\mathrm{SCCmec):} \mathrm{guidelines} \mathrm{for}$ reporting novel SCCmec elements. Antimicrob Agents Chemother 2009, 53:4961-4967.

20. Chongtrakool P, Ito T, Ma XX, Kondo Y, Trakulsomboon S, Tiensasitorn C, Jamklang M, Chavalit T, Song JH, Hiramatsu K: Staphylococcal cassette chromosome mec (SCCmec) typing of methicillin-resistant Staphylococcus aureus strains isolated in 11 Asian countries: a proposal for a new nomenclature for SCCmec elements. Antimicrob Agents Chemother 2006, 50:1001-1012.

21. Jansen WTM, Beitsma MM, Koeman CJ, van Wamel WJB, Verhoef J, Fluit AC: Novel mobile variants of staphylococcal cassette chromosome mec in Staphylococcus aureus. Antimicrob Agents Chemother 2006, 50:2072-2078

22. Mongkolrattanothai K, Boyle S, Murphy TV, Daum RS: Novel non-mecAcontaining staphylococcal chromosomal cassette composite island containing $p b p 4$ and tagF genes in a commensal staphylococcal species: a possible reservoir for antibiotic resistance islands in Staphylococcus aureus. Antimicrob Agents Chemother 2004, 48:1823-1836.

23. Baba T, Bae T, Schneewind O, Takeuchi F, Hiramatsu K: Genome sequence of Staphylococcus aureus strain Newman and comparative analysis of staphylococcal genomes: polymorphism and evolution of two major pathogenicity islands. J Bacteriol 2008, 190:300-310.

24. Parks AR, Peters JE: Transposon $T n 7$ is widespread in diverse bacteria and forms genomic islands. J Bacterio/ 2007, 189:2170-2173.

25. Chandler M, Fayet O: Translational frameshifting in the control of transposition in bacteria. Mol Microbiol 1993, 7:497-503.

26. Waldron DE, Lindsay JA: Sau1: a novel lineage-specific type I restrictionmodification system that blocks horizontal gene transfer into 
Staphylococcus aureus and between S. aureus isolates of different lineages. J Bacterio/ 2006, 188:5578-5585.

27. Bestebroer J, Poppelier MJJG, Ulfman LH, Lenting PJ, Denis CV, van Kessel KPM, van Strijp JAG, de Haas CJC: Staphylococcal superantigen-like 5 binds PSGL-1 and inhibits P-selectin-mediated neutrophil rolling. Blood 2007, 109:2936-2943.

28. Langley R, Wines B, Willoughby N, Basu I, Proft T, Fraser JD: The staphylococcal superantigen-like protein 7 binds IgA and complement C5 and inhibits IgA-Fc alpha Rl binding and serum killing of bacteria. $J$ Immunol 2005, 174:2926-2933.

29. Makris G, Wright JD, Ingham E, Holland KT: The hyaluronate lyase of Staphylococcus aureus - a virulence factor? Microbiology 2004, 150:2005-2013.

30. Hynes WL, Walton SL: Hyaluronidases of Gram-positive bacteria. FEMS Microbiol Lett 2000, 183:201-207.

31. Higuchi W, Takano T, Teng L, Yamamoto T: Structure and specific detection of staphylococcal cassette chromosome mec type VII. Biochem Biophys Res Commun 2008, 377:752-756.

32. Black CC, Solyman SM, Eberlein LC, Bemis DA, Woron AM, Kania SA: Identification of a predominant multilocus sequence type, pulsed-field gel electrophoresis cluster, and novel staphylococcal chromosomal cassette in clinical isolates of mecA-containing, methicillin-resistant Staphylococcus pseudintermedius. Vet Microbiol 2009, 139:333-338.

33. Katayama $Y$, Ito T, Hiramatsu K: A new class of genetic element, staphylococcus cassette chromosome mec, encodes methicillin resistance in Staphylococcus aureus. Antimicrob Agents Chemother 2000, 44:1549-1555.

34. Hasman $\mathrm{H}$, Aarestrup FM: tcrB, a gene conferring transferable copper resistance in Enterococcus faecium: occurrence, transferability, and linkage to macrolide and glycopeptide resistance. Antimicrob Agents Chemother 2002, 46:1410-1416.

35. Enright MC, Robinson DA, Randle G, Feil EJ, Grundmann H, Spratt BG: The evolutionary history of methicillin-resistant Staphylococcus aureus (MRSA). Proc Natl Acad Sci USA 2002, 99:7687-7692.

36. Zhu W, Clark NC, McDougal LK, Hageman J, McDonald LC, Patel JB: Vancomycin-resistant Staphylococcus aureus isolates associated with Inc18-like vanA plasmids in Michigan. Antimicrob Agents Chemother 2008, 52:452-457.

37. Sung JML, Lindsay JA: Staphylococcus aureus strains that are hypersusceptible to resistance gene transfer from enterococci. Antimicrob Agents Chemother 2007, 51:2189-2191.

38. Abajy MY, Kopec J, Schiwon K, Burzynski M, Doring M, Bohn C, Grohmann E: A type IV-secretion-like system is required for conjugative DNA transport of broad-host-range plasmid pIP501 in gram-positive bacteria. J Bacterio/ 2007, 189:2487-2496.

39. Kadlec K, Ehricht R, Monecke S, Steinacker U, Kaspar H, Mankertz J, Schwarz S: Diversity of antimicrobial resistance pheno- and genotypes of methicillin-resistant Staphylococcus aureus ST398 from diseased swine. J Antimicrob Chemother 2009, 64:1 156-1164

40. Herron-Olson L, Fitzgerald JR, Musser JM, Kapur V: Molecular correlates of host specialization in Staphylococcus aureus. PLOS ONE 2007, 2:e1120.

41. Rooijakkers SHM, Ruyken M, Roos A, Daha MR, Presanis JS, Sim RB, van Wamel WJB, van Kessel KPM, van Strijp JAG: Immune evasion by a staphylococcal complement inhibitor that acts on C3 convertases. Nat Immunol 2005, 6:920-927.

42. Bjerketorp J, Nilsson M, Ljungh A, Flock Jl, Jacobsson K, Frykberg L: A novel von Willebrand factor binding protein expressed by Staphylococcus aureus. Microbiology 2002, 148:2037-2044.

43. Kroh HK, Panizzi P, Bock PE: Von Willebrand factor-binding protein is a hysteretic conformational activator of prothrombin. Proc Natl Acad Sci USA 2009, 106:7786-7791.

44. Bjerketorp J, Jacobsson K, Frykberg L: The von Willebrand factor-binding protein (vWbp) of Staphylococcus aureus is a coagulase. FEMS Microbiol Lett 2004, 234:309-314.

45. Welinder-Olsson C, Floren-Johansson K, Larsson L, Oberg S, Karlsson L, Ahren C: Infection with Panton-Valentine leukocidin-positive methicillin-resistant Staphylococcus aureus t034. Emerg Infect Dis 2008, 14:1271-1272. doi: 10.1186/1471-2164-11-376

Cite this article as: Schijffelen et al., Whole genome analysis of a livestockassociated methicillin-resistant Staphylococcus aureus ST398 isolate from a case of human endocarditis BMC Genomics 2010, 11:376

\section{Submit your next manuscript to BioMed Central and take full advantage of:}

- Convenient online submission

- Thorough peer review

- No space constraints or color figure charges

- Immediate publication on acceptance

- Inclusion in PubMed, CAS, Scopus and Google Scholar

- Research which is freely available for redistribution

Submit your manuscript at www.biomedcentral.com/submit
C) Biomed Central 